\title{
Die Variolation im Spiegel der Korrespondenz Albrecht von Hallers (1708-1777) mit Achilles Mieg (1731-1799)
}

\author{
Von Marie-Louise Portmann
}

Die Pocken, eine in der Alten Welt schon seit frühester Zeit bekannte Krankheit, waren besonders virulent im 18. Jahrhundert, in der Zeit, in der Albrecht von Haller lebte. Der Basler Arzt und Mathematiker Daniel Bernoulli (1700-1782), der sich eingehend mit der Mortalität und Morbidität dieser Krankheit befaßte, errechnete auf Grund eines großen Zahlenmaterials aus den Städten Paris und London, daß 1/13 einer Generation an den Pocken starb ${ }^{1}$. Ein neuerer Forscher, Enrique Paschen (1860-1936), Professor für Impftechnik in Hamburg, kommt zum selben Ergebnis, wenn er im «Handbuch der pathogenen Mikroorganismen», hrsg. von W. Kolle, R. Kraus und P. Uhlenhuth, schreibt, daß in früheren Zeiten $1 / 14$ bis $1 / 12$ aller Todesfälle durch Blattern verursacht war ${ }^{2}$.

Sehr früh schon wußte man sich in China und Indien durch die Technik der Variolation, auch Inokulation genannt, gegen die Pocken zu schützen. Diese bestand darin, daß man, wie in China, getrockneten Pockeneiter in die Nase blies und so eine mildere Form der Krankheit hervorrief, die dann Immunität erzeugte, oder daß man, wie in Indien, den Impfstoff durch Einritzungen in die Haut einführte $^{3}$. Im 18. Jahrhundert war die Variolation auch in der Türkei seit langem bekannt, wo sie griechische Ärzte ${ }^{4}$ sowie Lady Mary Wortley Montagu (1690-1762), die Gattin des britischen Botschafters in Konstantinopel, kennenlernten. Lady Montagu ließ ihre eigenen Kinder inokulieren, und nach ihrer Rückkehr nach England 1721 gelang es ihr, das Interesse des britischen Königshauses für die neue Methode zu gewinnen, worauf die Kunde von der neuen Möglichkeit, sich gegen die Pocken zu schützen, bald in eine weitere Öffentlichkeit drang ${ }^{5}$.

Bei Variolation verliefen die Pocken gewöhnlich mild, doch kam es immer wieder vor, daß gewisse Individuen schwer daran erkrankten, erblindeten oder sogar starben. Es bestand auch die Gefahr, daß die Variolation eine echte Pockenepidemie unter den nicht geimpften Individuen auslösen konnte. Dies alles wurde schon früh erkannt und brachte es mit sich, daß in der zweiten Hälfte des 18. Jahrhunderts eine eigentliche Kontroverse zwischen Befürwortern und Gegnern der Variolation im Gange war. Es ist bemerkenswert, daß namentlich bei Vertretern der gebildeteren Gesellschaftsschicht die neue Methode raschen Eingang fand, was auf die aufgeschlossene Geisteshaltung im Zeitalter der Aufklärung zurückzuführen ist. 
Es war ein Schweizer Arzt, der Genfer Théodore Tronchin (1709-1781), welcher als Praktiker 1748 in Amsterdam die erste Inokulation auf dem Kontinent an seinem eigenen Sohn vornahm ${ }^{6}$. Tronchins Beispiel machte Schule in Genf, wo der Chirurg Daniel Guyot (1704-1780) im September 1750 der 18jährigen Mademoiselle Gallatin-Tronchin die Pocken einpfropfte. Es war dies die erste Variolation in der Schweiz ${ }^{7}$. Die neue Methode wurde in der Rhonestadt jedoch erst 1751 offiziell gestattet, nachdem die unehelichen Kinder, die sich im Genfer Spital befanden, erfolgreich inokuliert worden waren. Dem Beispiel Englands war man auch hier gefolgt, denn König Georg I. hatte die Variolation erst gestattet, nachdem der Eingriff an sechs Sträflingen mit Erfolg vorgenommen worden war und nachdem auch sechs Waisenkinder die Probe bestanden hatten ${ }^{8}$. Von Genf aus gelangte die Inokulation nach Lausanne, wo sie in dem Arzt Samuel-AugusteAndré-David Tissot (1728-1797), dem Freund Albrecht von Hallers, einen eifrigen Befürworter fand. Tissot berichtet, daß im Herbst 1753 eine Dame in Lausanne eigenhändig ihren Sohn inokuliert habe, und dies mit gutem Erfolg. Im folgenden Jahr habe dann der aus Genf herbeigeholte Chirurg Guyot weitere Kinder inokuliert, denn Guyot besaß bereits reiche Erfahrung auf diesem Gebiet, hatte er doch seit 1750 die Pocken schon etwa 40 Personen eingepfropft ${ }^{9} .1754$ erschien dann auch in Lausanne Tissots erste Schrift über die Variolation, betitelt «L'inoculation justifiée», die eine Apologie der neuen Methode beinhaltet.

In Basel hatten vor allem die Brüder Daniel und Johannes II Bernoulli (1710 bis 1790) von den Vorteilen der Pockenimpfung Kenntnis und waren bestrebt, diese in Basel einzuführen. Johannes Bernoulli war bereit, seine eigenen Söhne variolieren zu lassen, wenn zuvor eine Probe gemacht worden sei. Der Arzt, der den Eingriff vornehmen sollte, war Achilles Mieg, der während vieler Jahre mit Albrecht von Haller korrespondierte, wobei er in vielen Briefen auf seine Erfahrungen mit der Variolation zu sprechen kam und auch seinen Berner Freund um dessen Meinung fragte.

Achilles Mieg wurde 1731 als Sohn des Chirurgen J. Peter Mieg in Basel geboren. Er genoß zunächst seine Ausbildung in Basel und wurde hier im Juni 1748 Magister artium. Später ging er nach Holland, wo er sich von 1752 bis 1755 als Arzt und Wundarzt der Soldaten und Stadtarmen in Maastricht betätigte. Dann kehrte er nach Basel zurück, um zum Dr.med. zu promovieren. Da er schon 1752 sein Examen gemacht hatte und seine Dissertation «De Flatibus» schon damals gedruckt worden war, stand seiner Promotion nichts im Wege. Doch schon vorher bat er die Medizinische Fakultät um Erlaubnis, die Variolation vornehmen zu dürfen. Nachdem auch die Theologen zugestimmt hatten, vollzog Mieg am 29. Mai 1756, fünf Tage vor seiner Promotion, die erste Inokulation in Basel an Christoph 
Meyer, dem Söhnlein eines Wagners. Nach erfolgreichem Verlauf dieser Probe ließ Johannes II Bernoulli seine beiden Knaben Emanuel und Daniel ebenfalls durch Mieg variolieren. Im folgenden Jahr 1757 wurden auch Bernoullis ältestem Sohn, dem 12 1/2 jährigen Johannes, die Pocken eingepfropft. Nach überstandener Krankheit hielt dieser seine Baccalaureats-Rede «De insitione variolarum», über das neue Impfverfahren zum Schutz gegen die Pocken ${ }^{10}$.

Achilles Mieg variolierte noch viele Kinder und Jugendliche und war bald ein gesuchter Praktiker in Basel. Von 1769 an war er Dozent für Chirurgie, populäre Medizin und Heilkräuterkunde für Landpfarrer an der Universität. Da in Basel das Los über die Ernennung zum Professor entschied und Mieg viermal Pech hatte, wurde er erst 1777 Inhaber des Lehrstuhls für praktische Medizin. Als solcher erwarb er sich besondere Verdienste um den klinischen Unterricht, und er blieb unternehmend bis zu seinem am 11. Dezember 1799 erfolgten Tode ${ }^{11}$.

Der Briefwechsel Hallers mit Mieg, der in lateinischer Sprache geführt wurde, ist sozusagen vollständig erhalten. Auf der Burgerbibliothek Bern werden 59 handschriftliche Briefe Miegs an Haller aufbewahrt, die den Zeitraum vom November 1755 bis Dezember 1777 umfassen ${ }^{12}$. 27 dieser Briefe sind ganz oder teilweise abgedruckt in den Bänden «Epistolarum ab eruditis viris ad Albertum Hallerum scriptarum Pars I», die von Haller selber in den Jahren 1773-1775 herausgegeben wurden ${ }^{13}$. Einer der gedruckten Briefe ist handschriftlich nicht mehr vorhanden. Die 59 Originalbriefe Hallers an Mieg, die den gleichen Zeitraum umfassen, sind in einem schönen Sammelband der Basler Universitätsbibliothek enthalten, den Friedrich Miescher-Rüsch (1844-1895), Professor der Physiologie und Pathologie, der Bibliothek am hundertsten Todestag Hallers, am 12. Dezember 1877, zum Geschenk übergeben hat ${ }^{14}$.

Die Korrespondenz beginnt mit einem Brief Miegs an Haller vom 5. November 1755, in welchem er berichtet, daß er sich studienhalber in Holland aufgehalten habe, wo ihm Professor Johann David Hahn (1729-1784) aus Utrecht ein Bücherpaket für Haller mitgegeben hatte. Mieg bietet Haller auch ein Verzeichnis derjenigen Pflanzen an, die in der Umgebung von Maastricht vorkommen, sowie Notizen über die Flora Basels ${ }^{15}$. In seiner Antwort vom 8. November 1755 bestätigt Haller den Empfang der Büchersendung Hahns und nimmt gerne die Offerte des jungen Baslers an, Haller Pflanzen und botanische Aufzeichnungen zu liefern. Als Gegengabe bietet der Berner Gelehrte Alpenpflanzen und andere Exemplare an, die er besitzt ${ }^{16}$. So nahm der Briefwechsel seinen Anfang, in welchem der Botanik eine überragende Stellung zukommt, was schon von H. Christ in den Verhandlungen der Naturforschenden Gesellschaft in Basel, Bd. 29, 1918, gewürdigt worden ist ${ }^{17}$. 


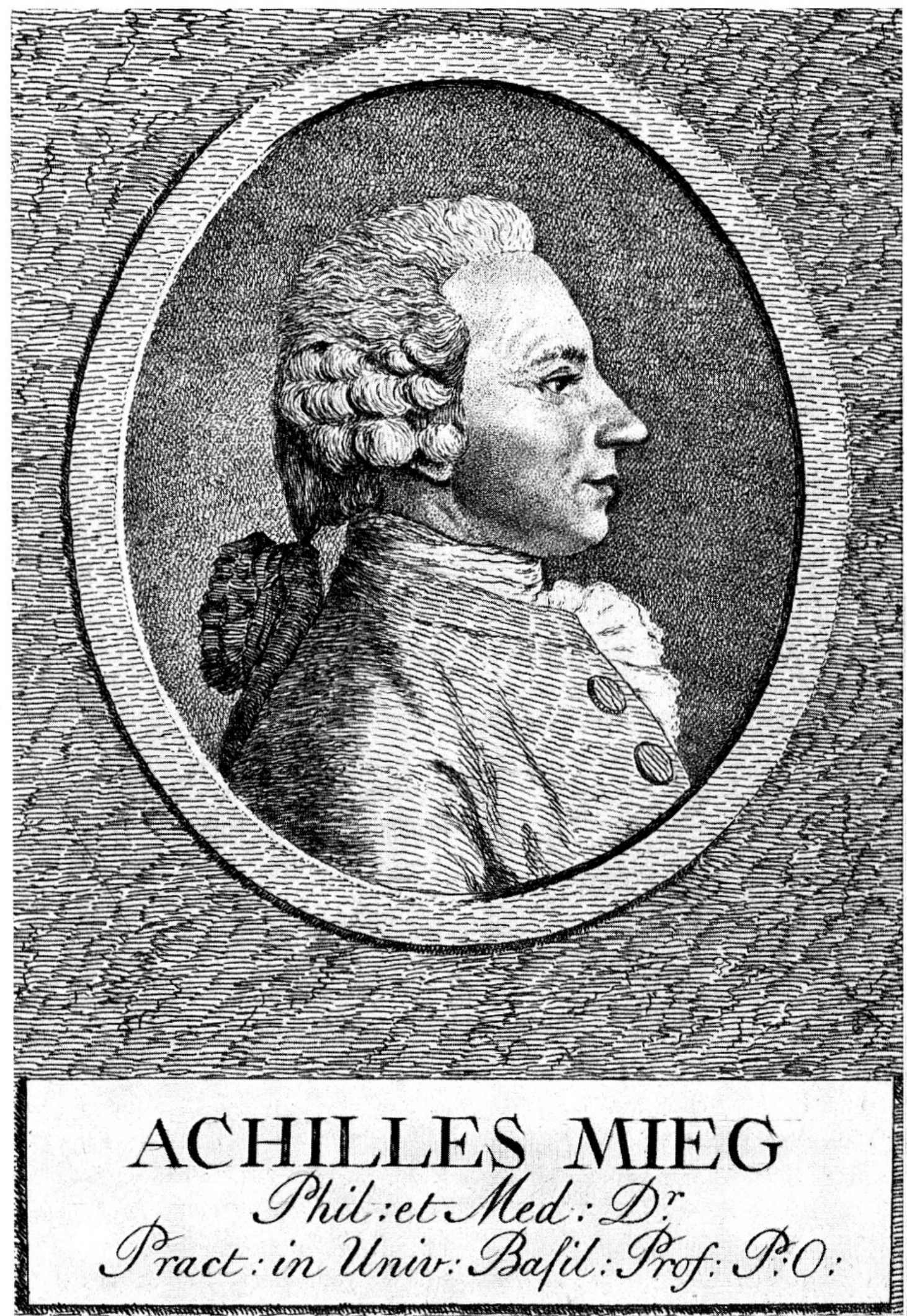


Den Interessen der beiden Korrespondenten entsprechend kommt aber auch die Variolation zur Sprache, und auf diese sei hier im besonderen eingegangen. Am 10. November 1756 schildert Mieg, wie auf Betreiben der Brüder Daniel und Johannes II Bernoulli die Inokulation in Basel eingeführt worden ist ${ }^{18}$. Die Details sind oben bereits erzählt worden. Schon am 8. Juni 1757 hat Mieg von sechs weiteren Variolationen zu berichten. Bei einem der jungen Patienten seien die Pocken nicht zum Ausbruch gekommen, aber desto reichlicher sei der Eiter geflossen, was Mieg zuversichtlich macht, daß auch dieses Kind inskünftig nicht an den Pocken erkranken werde, denn von einem ebenfalls inokulierten Schwesterchen sei es nicht angesteckt worden. Unter den Variolierten Miegs war auch der älteste Sohn des Mathematikers Johannes II Bernoulli, dessen bereits erwähnte Baccalaureats-Rede Mieg seinem Briefe beilegt. Haller druckte diese Oratio dann zusammen mit den Briefen Miegs ab in den an ihn gerichteten Epistolae ${ }^{19}$. Mieg hat auch gehört, daß Haller seine Tochter varioliert habe. Es war dies die erste Inokulation in Bern. Der Basler möchte wissen, wie der Erfolg gewesen sei. Hallers Antwort ist datiert vom 11. Juni $1757^{20}$. Er berichtet, daß seine älteste Tochter mit gutem Erfolg geimpft worden sei. Es handelte sich dabei um die erste Tochter aus Hallers dritter Ehe mit Sophie Teichmeyer. Das Mädchen hieß Amalia Catharina (genannt Emilie) und wurde im selben Jahr 1757 mit ihrem um 20 Jahre älteren entfernten Vetter Samuel Haller vermählt ${ }^{21}$. Als die Pocken bei Hallers Tochter nicht ausbrechen wollten, wurde sie zweimal mit neuem Pockeneiter von andern Personen behandelt, doch erschienen keine Pusteln. Da die Wunden jedoch schön eiterten, gab man sich zufrieden und hielt die Tochter für immun.

Hier muß ein Wort über die in der Schweiz gebräuchliche Technik der Variolation eingeflochten werden. Man machte Einschnitte an beiden Oberarmen und erzeugte dann die Infektion, indem man einen Faden in die Schnitte einführte, der mit dem Eiter von Pockenpusteln getränkt und dann getrocknet worden war ${ }^{22}$. Nach etwa zehn Tagen brachen die Pocken aus, aber meist war der Verlauf milder als bei Ansteckung auf natürlichem Wege. Es gab indessen stets Individuen, die bereits immun waren, wie z. B. Hallers Tochter. In einem Brief vom 18. Oktober 1757 berichtet Mieg von einem ähnlichen Fall und erkundigt sich, wie Haller zum Erfolg gekommen sei $^{23}$. In seiner Antwort vom 22. Oktober 1757 berichtet Haller, daß die Einschnitte bei seiner Tochter während sechs Wochen geeitert hätten. Zweimal habe man in die offenen Wunden neuen Pockeneiter eingeführt. Einen neuen Einschnitt habe er indessen nicht gemacht ${ }^{24}$.

Am 16. Januar 1764 möchte Haller Bescheid wissen über Miegs neue Erfahrungen mit der Variolation. Daher verweist der jetzt viel beschäftigte Basler 
Arzt am 11. September desselben Jahres auf seinen ausführlichen Bericht, den er in Form eines Briefes an den Zürcher Arzt Salomon Schinz (1734-1784) im vorigen Jahr erstattet habe und der in den Abhandlungen der Zürcher Naturforschenden Gesellschaft abgedruckt werde. Im Jahre 1766 erschien dann diese Schrift Miegs in den genannten Abhandlungen ${ }^{25}$ zusammen mit weiteren Briefen deutschschweizerischer Ärzte über ihre Erfahrungen mit der Variolation. Dieser Band gewährt uns Einblick in alle Details des Vorgehens bei der neuen Impfmethode. Miegs Beitrag ist in dieser Arbeit der weitaus umfangreichste.

Im Jahre 1765 erkundigte sich der Franzose Charles-Marie de La Condamine (1701-1774) in Basel nach den Erfolgen der Variolation in der Schweiz ${ }^{26}$. Dieser Gelehrte, der vor allem als Mathematiker und Forschungsreisender bekannt ist, hatte schon 1754 einen epochemachenden Aufsatz zur Geschichte und Apologie der Blatternimpfung geschrieben ${ }^{27}$ und bereitete nun eine neue Schrift vor, die 1773 unter dem Titel «Histoire de l'inoculation de la petite vérole» erschien. In seinem Brief vom 10. September 1765 bittet Mieg seinen Berner Freund, entweder Herrn de La Condamine selbst oder ihm, Mieg, die Städte im Berner Gebiet zu nennen, wo inokuliert wurde, und die Namen der Variolierten und deren Zahl anzugeben sowie den Erfolg oder Mißerfolg zu melden ${ }^{28}$. In seiner Antwort vom 29. September 1765 berichtet dann Haller dem Basler Arzt von den Inokulationen, von denen er Kenntnis hat: er selbst habe seine Tochter geimpft, wobei keine Pockenerkrankung aufgetreten sei. Sodann habe er die Söhne der Herren Karl Emanuel von Bonstetten (1706-1773) und Johann Bernhard von Muralt (1709 bis 1780 ) mit Erfolg varioliert. Der Berner Arzt Dr. Wilhelm Hilfer habe an zwei Schwestern de Mollens den Eingriff vorgenommen. Auch der Chirurgus Küffer habe in Hallers Gegenwart drei Söhne des Herrn Abraham Ahasverus von Tscharner (1711-1767) erfolgreich geimpft. In Lausanne inokuliere Dr. Tissot und in Vevey der Chirurg Rochat. Über das weitere weiß Haller nicht Bescheid, doch anerbietet er sich, auf Wunsch des Herrn de La Condamine an Rochat zu schreiben, und von Dr. Hilfer könne er noch mehr erfahren ${ }^{29}$.

Zu Ende der sechziger Jahre wird der Briefwechsel zwischen Haller und Mieg spärlich, um dann eine Zeitlang ganz aufzuhören. Den Grund nennt Mieg in seinem Brief vom 12.Januar 1768. Der Basler Arzt hat die Beschäftigung mit der Botanik seit einigen Jahren ganz aufgeben müssen und muß nun auch seine Korrespondenz einstellen, da ihn seine Praxis vollkommen absorbiert ${ }^{30}$. Den Briefwechsel führte der Basler Botaniker Werner de La Chenal (1736-1800) weiter ${ }^{31}$.

Erst am 10. Mai 1774 nahm Haller die Korrespondenz mit Mieg wieder auf mit der Schilderung seiner Krankheitssymptome ${ }^{32}$. Von da an wurde Hallers jüngerer Basler Freund zu einem treuen Berater während seines Leidens. Der 
Hauptgegenstand des Briefwechsels ist denn auch Hallers Krankheitsgeschichte. Nur in einem einzigen Brief kommt Mieg noch einmal auf die Variolation zu sprechen $^{33}$, worauf Haller antwortet, daß er die Methode des Baslers billige ${ }^{34}$. In seinem letzten Brief an Mieg vom 3. Dezember 1777 gratuliert der vom Tode gezeichnete Haller seinem Basler Freund zu der endlich erlangten Professur für praktische Medizin und gibt seiner Überzeugung Ausdruck, daß in Mieg ein würdiger Nachfolger der Platter und der Bauhin gefunden sei ${ }^{35}$. Haller starb am 12. Dezember 1777.

Die Variolation blieb immer eine umstrittene Methode des Pockenschutzes, bis Edward Jenner (1749-1823) 1796 die erste Kuhpockenimpfung und der experimentelle Nachweis der dadurch bewirkten Immunität gegen Pocken gelang ${ }^{36}$. Damit wurde die Variolation durch die Vakzination abgelöst.

In England jedoch wurde die Variolation noch 1841 ausgeübt, da sie dort die meisten Anhänger hatte. Eine Parlamentsakte mußte schließlich diese Methode der Pockenbekämpfung verbieten, da sie eine Gefahr darstellte, indem bei Variolation neue Epidemien ausbrechen konnten ${ }^{37}$.

Durch die Bemühungen der Weltgesundheitsorganisation, die großangelegte Impfungen durchführte in jenen Gegenden der Welt, wo die Pocken endemisch waren, gelingt es nun, die Pocken zum Aussterben zu bringen. Die Beamten dieser Organisation sind daran, in den abgelegenen Gebieten Äthiopiens die ganze Bevölkerung durchzuimpfen, und wenn dieses Ziel erreicht ist, wird die Menschheit von den Pocken befreit sein.

NB. Der Band «Albrecht von Hallers Briefe an Auguste Tissot», herausgegeben von Erich Hintzsche, Bern/Stuttgart/Wien 1977, ist erst nach Abschluß dieses Manuskripts erschienen. Auch in dieser Korrespondenz ist öfters von der Variolation die Rede, doch bedarf das hier Mitgeteilte keiner Ergänzung. 


\section{Anmerkungen}

${ }^{1}$ Bernoulli, Daniel, Essai d'une nouvelle analyse de la mortalité causée par la petite vérole et des avantages de l'inoculation pour la prévenir. Histoire de l'Académie royale des Sciences etc., Paris 1760, S.1-81. Mém. de l'Académie royale des Sciences, Paris 1760, S.1-45. Huber, Friedrich, Daniel Bernoulli (1700-1782) als Physiologe und Statistiker. Basler Veröffentlichungen zur Geschichte der Medizin und der Biologie, Fasc. VIII, Basel 1959, S. $78 \mathrm{ff}$.

${ }^{2}$ Paschen, Enrique, Pocken. In: Handbuch der pathogenen Mikroorganismen. Hrsg. W.Kolle, R.Kraus, P. Uhlenhuth, Bd. 8, 2.Teil., Jena/Berlin/Wien 1930, S. 824.

${ }^{3}$ Ackerknecht, Erwin H., Geschichte und Geographie der wichtigsten Krankheiten, Stuttgart 1963, S. 55-60.

Parish, H. J., Victory with Vaccines, Edinburgh and London 1968, S. 8.

4 Timoni, Emanuel (gest.1718), An account, or history, of the procuring of the smallpox by incision or inoculation, as it has for some time been practised at Constantinople. Phil. Trans. 29 (1714-16), S. 72-82.

Pylarini, Giacomo (1659-1718), Nova et tuta variolas excitandi per transplantationem methodus, nuper inventa et in usum tracta. Phil.Trans.29 (1714-16), S. 393-399.

5 Parish, 1.c., S. 9-10.

${ }^{6}$ Ackerknecht, Erwin H., und Buess, Heinrich, Kurze Geschichte der großen Schweizer Ärzte, Bern/Stuttgart/Wien 1975, S. 38.

7 Gautier, Léon, La Médecine à Genève jusqu'à la fin du dix-huitième siècle, Genève 1906, S. $390-392$.

8 Paschen, E., l.c., S. 826.

9 Olivier, Eugène, Médecine et Santé dans le pays de Vaud au XVIII ${ }^{e}$ siècle, Tome II, Lausanne 1939, S. 681.

10 Burckhardt, Albrecht, Geschichte der Medizinischen Fakultät zu Basel 1460-1900, Basel 1917, S. 234-236.

Christ, H., Der Briefwechsel der Basler Botaniker des 18. Jahrhunderts Achilles Mieg, Werner de La Chenal und Jacob Christoph Ramspeck mit Albrecht von Haller. Verhandlungen der Naturforschenden Gesellschaft in Basel 29 (1918), S.1-32.

Staehelin, Andreas, Geschichte der Universität Basel 1632-1818, 2.Teil, Basel 1957, S. 559.

11 Burckhardt, Albrecht, l.c., S. 256.

12 Herr Dr.Hans A.Haeberli von der Burgerbibliothek Bern hatte die Freundlichkeit, mir Photokopien der Briefe Achilles Miegs herstellen zu lassen. Auch den Beamten der Handschriftenabteilung der Universitätsbibliothek Basel bin ich zu Dank verpflichtet.

${ }_{13}$ Haller, Albrecht, Epistolae ab eruditis viris ad Alb. Hallerum, vol. IV-VI, Bern 1773/1775.

14 Christ, H., l.c., S. 1 .

15 Burgerbibliothek Bern, Mss. Hist. Helv. XVIII. 14, S.177.

16 Universitätsbibliothek Basel, Ms. G² II 82, Nr.1.

17 Christ, H., l. c., S. 14-32.

18 Burgerbibliothek Bern, Mss. Hist. Helv. XVIII. 15, S.127.

Epistolarum ab eruditis viris ad Albertum Hallerum scriptarum Pars I, vol. IV, S. 44-47. 
19 Burgerbibliothek Bern, Mss. Hist. Helv. XVIII. 16, S. 82.

Epistolarum ab eruditis viris ad Albertum Hallerum scriptarum Pars I, vol. IV, S. 83-93. Burckhardt, Fritz (Hrsg.), Autobiographie des Johannes II Bernoulli. Basler Zeitschrift für Geschichte und Altertumskunde 6 (1906), S. 304: "Gegen den Herbst 1756 ließ ich meinen 2 Söhnen Emanuel und Daniel die Kindsblattern einpfropfen, welche operation vorher hier in Basel noch an niemand als eines Wagners Kind ware gemacht worden. Sie hatte, Gott seye danck, einen erwünschten success. Seither habe ich diese operation mit dem nämlichen Erfolg sowohl auf meinem ältesten als auch hernach auf meinem jüngsten Sohn thun lassen.»

20 Universitätsbibliothek Basel, Ms. G II 82, Nr. 6.

${ }^{21}$ Beer, Rüdiger Robert, Der große Haller, Säckingen 1947, S. 98.

22 Mieg, Achilles, Von dem Erfolg der Einpfropfung der Pocken an einigen Orten in unserer Schweiz. Abhandlungen der Naturforschenden Gesellschaft in Zürich 3 (1766), S. 66.

${ }^{23}$ Burgerbibliothek Bern, Mss. Hist. Helv. XVIII. 16, S.149.

Epistolarum ab eruditis viris ad Albertum Hallerum scriptarum Pars I, vol. IV, S.143.

${ }^{24}$ Universitätsbibliothek Basel, Ms. G' II 82, Nr. 10.

${ }_{25}$ Universitätsbibliothek Basel, Ms. G ${ }^{2}$ II 82, Nr. 32.

Burgerbibliothek Bern, Mss. Hist. Helv. XVIII. 23, S.131.

Epistolarum ab eruditis viris ad Albertum Hallerum scriptarum Pars I, vol. V, S. 206-207. Von dem Erfolg der Einpfropfung der Pocken an einigen Orten in unserer Schweiz. $A b$ handlungen der Naturforschenden Gesellschaft in Zürich 3 (1766), S. 24-176.

${ }^{26}$ Charles Marie de La Condamine unterhielt einen regen Briefwechsel mit Daniel Bernoulli. Universitätsbibliothek Basel L I a 685.

27 Trois Mémoires sur l'inoculation 1754, 1758, 1765.

Lettre à Mr. Maty sur l'état présant de l'inoculation en France. Mém. de l'Acad. des sc. de Paris 1764. Vgl. Biographisches Lexikon der hervorragenden Ärzte aller Zeiten und Völker, hrsg. August Hirsch, 2. Aufl., Berlin/Wien 1930, Bd. 2, S. 89, sowie Biographie universelle, tome IX, S. 6-8.

${ }^{28}$ Burgerbibliothek Bern, Mss. Hist. Helv. XVIII. 24, S. 113.

Epistolarum ab eruditis viris ad Albertum Hallerum scriptarum Pars I, vol. V, S. 233-242.

${ }^{29}$ Universitätsbibliothek Basel, Ms. G ${ }^{2}$ II $82, \mathrm{Nr} .37$.

${ }^{30}$ Burgerbibliothek Bern, Mss. Hist. Helv. XVIII. 28, S. 12.

${ }^{31}$ Christ, H., l.c., S. 28.

32 Universitätsbibliothek Basel, Ms. G² II 82, Nr. 39.

${ }^{33}$ Brief Miegs an Haller vom 10. Juni 1775. Burgerbibliothek Bern, Mss. Hist. Helv. XVIII. 35, S. 109.

${ }^{34}$ Brief Hallers an Mieg vom 14.Juni 1775. Universitätsbibliothek Basel, Ms. G² II 82, Nr. 50.

35 Universitätsbibliothek Basel, Ms. G² II 82, Nr. 59.

${ }^{36}$ Koelbing, Huldrych M., Im Kampf gegen Pocken, Tollwut, Syphilis. Gute Schriften, Basel 1974.

${ }^{37}$ Dies schreibt der französische Kliniker Armand Trousseau (1801-1867), der die Inokulation noch dann anwendete, wenn ihm der Impfstoff ausgegangen war.

Clinique médicale de l'Hôtel Dieu de Paris, 2. Bd., 1861. Ed. par A.Vannotti, S. Cruchaud et Georges-A. Du Bois, Genève/Paris/Bruxelles 1963, vol. I, S. 133 f. 


\section{Summary}

In the eighteenth century smallpox was very virulent in Europe. This was just the period when variolation was introduced into England from Eastern countries, where it had been practised for a very long time. Lady Mary Wortley Montagu, who was the wife of the British Ambassador in Constantinople made it known in England. In 1748 the Geneva physician Théodore Tronchin (1709-1781) performed the first inoculation on the continent in Amsterdam. Soon the surgeon Daniel Guyot (1704-1780) imitated him in his native Geneva, whence Auguste Tissot (1728-1797) of Lausanne made the acquaintance with the new method of preventing smallpox. In Basel the brothers Daniel (1700-1782) and Johannes Bernoulli II (1710-1790) were eager in paving the way for variolation. Achilles Mieg, physician in Basel, inoculated the sons of Johannes Bernoulli II and other children in 1756 and 1757.

At the same time the correspondence between Achilles Mieg and Albrecht von Haller had begun, and as Haller too was interested in variolation their letters frequently dealt with this subject. Haller was the first to perform an inoculation in Bern, when he tried to immunize his own daughter Amalia Catharina. But as no pustules appeared, variolation was repeated but did not appear to be successful because the girl already was immune. In 1765 the French explorer Charles Marie de La Condamine (1701-1774) asked for information about variolation in Switzerland, which Mieg tried to give him through his friend Haller. The experience of Mieg in variolating children is reported in the "Abhandlungen der Naturforschenden Gesellschaft Zürich», Vol. 3, 1766.

After 1768, when Mieg had too many patients and no longer had the time to botanize his correspondence with Haller dropped. But it was started again by Haller in 1774 when he fell ill. Mieg was a staunch counsellor to this friend during the whole period of his disease. In his last letter, on December 3, 1777 the dying Haller congratulated his correspondent who had finally become Professor of Practical Medicine in Basel. Variolation was superseded by vaccination when Edward Jenner (1749-1823) succeeded in 1796 in inoculating cowpox as a prophylactic against smallpox.

Dr. Marie-Louise Portmann

Medizinhistorische Bibliothek

Schönbeinstraße 40

4056 Basel 